\title{
Riesgo perioperatorio del bypass gástrico reseccional en pacientes con obesidad mórbida. Estudio prospectivo de 684 pacientes
}

\author{
Attila Csendes J, Patricio Burdiles P, Ana María Burgos L, \\ Juan Carlos Díaz J, Italo Braghetto M, Fernando Maluenda G, \\ Jorge Rojas C, G uillermo W atkins S.
}

Perioperative risk among morbid obese patients subjected to gastric bypass

Background: Bariatric surgery is a complex procedure not exempt of complications. Aim: To assess mortality and complications of excisional gastric bypass among morbidly obese subjects. Material and methods: Prospective analysis of 684 morbid obese patients (age range 14-70 years, 525 females) subjected to an excisional gastric bypass. Major postoperative complications and mortality were registered. Results: Mean body mass index (BMI) of the subjects was $43.7 \mathrm{~kg} / \mathrm{m}^{2}$. One hundred sixty two patients had a BMI between 35 and $39.9 \mathrm{~kg} / \mathrm{m}^{2}, 419$ had a BMI between 40 and $49.9 \mathrm{~kg} / \mathrm{m}^{2}$ and 103 had a BMI over $50 \mathrm{~kg} / \mathrm{m}^{2}$. Two patients with a BMI of 52 and $56 \mathrm{~kg} / \mathrm{m}^{2}$ respectively, died in the postoperative period $(0.3 \%)$. Thirty six patients had major complications. Anastomotic fistula was the most common complication in 12 patients $(1.7 \%)$. Fourteen patients required a new operation due to complications. None of these died. The mean operative volume of the surgical team was 124 patients per year. Conclusions: Excisional gastric bypass has a low rate of mortality and complications, if the surgical team operates a large volume of patients (Rev Méd Chile 2006; 134: 849-54).

(Key w ords: Bariatric surgery; Gastric bypass; Obesity, morbid)

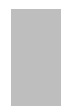

Recibido el 10 de junio, 2005. Aceptado el 12 de enero, 2006.

Departamento de Cirugía, Hospital Clínico de la

Universidad de Chile, Santiago Chile.

$\mathrm{E}^{1}$ tratamiento quirúrgico de pacientes con obesidad mórbida tiene significativamente mejores resultados en cuanto a baja de peso,

Correspondencia a: Attila Csendes, MD, FACS (Hon). Director Departamento de Cirugía, Hospital Clínico JJ Aguirre. Santos Dumont 999, Santiago Chile. Fono: 56-2-7774387. Fax: 56-2-7775043. E mail: acsendes@machi.med.uchile.cl mejoría de las comorbilidades y de la calidad de vida, comparado con pacientes no operados ${ }^{1,2}$.

La reducción de la mortalidad en pacientes operados, comparados con no operados, en dos seguimientos a largo plazo fue 75 y $90 \% 1,2$. Sin embargo, la cirugía en estos pacientes es un procedimiento de gran envergadura, con potenciales complicaciones y mortalidad operatoria, por 
lo que debe ser realizada por centros con gran experiencia en cirugía gástrica, lo que facilita y hace más expeditos los procedimientos quirúrgicos y optimiza el manejo de las complicaciones.

El objetivo del presente estudio fue analizar las cifras de mortalidad operatoria y la frecuencia de complicaciones intra o postoperatorias mayores en un centro dedicado a cirugías de alta complejidad, con experiencia muy importante en cirugía gástrica, que realiza más de 100 operaciones por año de cirugía bariátrica ${ }^{3}$.

\section{MATERIAL Y MÉTODO}

Pacientes. El grupo corresponde a 684 pacientes con obesidad mórbida, que ingresaron a un protocolo prospectivo especialmente diseñado para estos pacientes, que comenzó el 9 de agosto de 1999 y se cerró el 10 de enero de 2005. Todos tenían obesidad mórbida, es decir, un índice de masa corporal (IMC) de más de $40 \mathrm{~kg} / \mathrm{m}^{2}$ o bien, más de $35 \mathrm{~kg} / \mathrm{m}^{2}$ y una o más comorbilidades como hipertensión arterial, diabetes tipo II, dislipidemia o problemas osteoarticulares severos ${ }^{4,5}$. Se excluyeron 13 pacientes que fueron reoperados por falla de una gastroplastia previa ${ }^{6}$. Tampoco se incluyeron los pacientes sometidos a cirugía laparoscópica, que comenzó en agosto de 2002 (128 pacientes), como aquellos pacientes sometidos a bypass gástrico abierto sin gastrectomía (55 pacientes).

Técnica quirúrgica. En todos los pacientes se realizó laparotomía media supraumbilical o subcostal bilateral en los hiperobesos. La técnica ha sido descrita en publicaciones previas ${ }^{4,5}$. Básicamente consiste en realizar una gastrectomía de 95 a 97\% (casi total), dejando un reservorio gástrico vertical de menos de $20 \mathrm{ml}$ de capacidad en la curva menor. La anastomosis gastroyeyunal se realizó en todos con un stapler circular № 25 (Tyco Healthcare). La sección del duodeno, así como la confección de la bolsa gástrica se realizó con stapler GIA 60 y $80 \mathrm{~mm}$ (Tyco Healthcare). El largo del asa alimentaria en Y-de-Roux varió entre 125 y $150 \mathrm{~cm}$. En todos se tomó una biopsia hepática y se realizó colecistectomía de rutina. Se dejaron 2 drenajes, a la derecha e izquierda de la anastomosis gastroyeyunal. La impermeabilidad de esta anastomosis se probó durante la cirugía con la inyección de azul de metileno a través de una sonda nasogástrica. En el periodo postoperatorio inmediato se empleó heparina 5.000 unidades c/ 8 ó 12 h subcutánea, así como cefazolina $1 \mathrm{~g}$ $\mathrm{c} / 8 \mathrm{~h}$ por $24 \mathrm{~h}$. Todos los pacientes quedaron en la Unidad de Tratamiento Intermedio por 1 a 2 días. Se realizó kinesiterapia respiratoria intensiva y la levantada fue precoz, entre 24 y 48 h después de la cirugía.

La analgesia epidural se retiró a las 72 h. Se comenzó la realimentación oral al 4 ํ día postoperatorio. En todos, se realizó un control radiológico de la anastomosis gastroyeyunal con bario diluido ente el $4^{\circ}$ y $5^{0}$ día postoperatorio. El alta hospitalaria ocurrió entre el $6^{0}$ y 70 día en cerca de $94 \%$ de los pacientes.

Cálculos estadísticos. Para los valores de significación estadística, se empleó el test exacto de Fisher y el Chi cuadrado, tomando un $\mathrm{p}<0,05$ como estadísticamente significativo.

\section{Definiciones}

a. Se consideró mortalidad operatoria la que ocurrió hasta 30 días después de la cirugía o la que ocurrió dentro de la hospitalización, independiente del tiempo desde la operación inicial.

b. Complicaciones precoces: se consideraron todas las complicaciones que ocurrieron hasta los 30 días desde la operación inicial, aun cuando el alta hospitalaria haya ocurrido al 7o u $8^{\text {o }}$ día postoperatorio. Se consideraron sólo las complicaciones mayores, es decir, los que representaron algún cambio en el tratamiento (por ejemplo una reoperación) o que prolongaron la estadía hospitalaria más allá de lo habitual.

\section{ReSUltados}

Del total de los 684 pacientes, 525 fueron mujeres $(76,7 \%)$ y 159 fueron hombres (23,3\%), con una relación mujer:hombre de 3,3. La edad promedio fue de $39,7 \pm 11,9$ años, con un rango entre 14 y 70 años. El índice de masa corporal (IMC) promedio fue $43,7 \mathrm{~kg} / \mathrm{m}^{2}$. Hubo 162 pacientes $(23,7 \%)$ con IMC menor a $40 \mathrm{~kg} / \mathrm{m}^{2}, 419$ pacientes $(61,2 \%)$ con 
IMC entre 40 y $49,9 \mathrm{~kg} / \mathrm{m}^{2}$ y 103 pacientes (15,1\%) con IMC $\geq 50 \mathrm{~kg} / \mathrm{m}^{2}$ (hiperobesos). En la Tabla 1 se señala la mortalidad y la tasa de complicaciones mayores en estos pacientes sometidos a bypass gástrico reseccional. Hubo 2 fallecidos, ambos pacientes hiperobesos (de 56 y $52 \mathrm{~kg} / \mathrm{m}^{2}$ ). Uno falleció a los 32 días por una sepsis secunda-

\section{Tabla 1. M ortalidad y morbilidad postoperatoria en 684 pacientes con obesidad mórbida sometidos a bypass gástrico reseccional}

\begin{tabular}{|lrr|}
\hline Complicaciones mayores & $\mathrm{n}$ & $\%$ \\
\hline Fístula gastroyeyunal & $12(1)$ & 1,75 \\
Colección abdominal & 5 & 0,7 \\
Hemorragia línea sutura & $5(1)$ & 0,7 \\
Necrosis yeyuno & $1(1)$ & 0,15 \\
Obstrucción intestinal & $5(5)$ & 0,7 \\
Trombosis mesentérica & $1(1)$ & 0,15 \\
Trombosis venosa profunda & $2(1)$ & 0,3 \\
Necrosis pared abdominal & $1(1)$ & 0,15 \\
Hemoperitoneo & $1(1)$ & 0,15 \\
Necrosis omento mayor & $1(1)$ & 0,15 \\
Fístula duodenal & $2(1)$ & 0,3 \\
\hline Mortalidad & 2 & 0,29 \\
\hline
\end{tabular}

( ) número de pacientes reoperados. ria a una fístula gastroyeyunal y el otro paciente a los 27 días, con una hipertermia maligna persis-

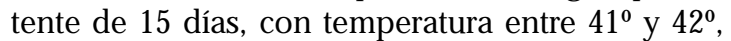
sin un foco séptico y sin ningún germen aislado en múltiples cultivos. La morbilidad mayor correspondió a 36 pacientes, es decir 5,3\%. Hubo 4 pacientes $(0,6 \%)$ en que la morbilidad fue probablemente secundaria a la gastrectomía: 2 fístulas duodenales, 1 hemoperitoneo y 1 necrosis del omento mayor. El resto de las complicaciones mayores son comunes a cualquier bypass gástrico. De los 12 pacientes con fístula gastroyeyunal, solo 1 paciente necesitó reoperación y los demás se manejaron conservadoramente. Hubo 5 pacientes con colecciones intraabdominales en los que no se comprobó la presencia de fístula y fueron manejados por radiología intervencional.

Hubo 5 pacientes con hemorragia precoz de la línea de sutura gastroyeyunal, entre el $1^{\mathrm{er}}$ y $8^{\circ}$ día postoperatorio. El primer paciente se reoperó, sin embargo, los demás fueron tratados y controlados endoscópicamente. Los 5 pacientes con obstrucción intestinal necesitaron una reoperación con evolución satisfactoria. La Tabla 2 señala la tasa de mortalidad y complicaciones mayores según el IMC. No hubo mortalidad en 581 pacientes con IMC bajo $50 \mathrm{~kg} / \mathrm{m}^{2}$. Hay una distribución similar de la prevalencia de las complicaciones, destacando la baja tasa en pacientes hiperobesos, pero sin diferencias significativas.

Tabla 2. M ortalidad y morbilidad de pacientes con obesidad mórbida sometidos a bypass gástrico resección según el índice de masa corporal. N = 684

\begin{tabular}{|c|c|c|c|}
\hline \multirow[t]{2}{*}{ Complicaciones } & \multicolumn{3}{|c|}{ Indice masa corporal kg/m² } \\
\hline & $\begin{array}{l}<40 \\
n=162\end{array}$ & $\begin{array}{l}40-49,9 \\
n=419\end{array}$ & $\begin{array}{l}\geq 50 \\
\mathrm{n}=103\end{array}$ \\
\hline Mortalidad & 0 & 0 & $2(1,94 \%)$ \\
\hline Fístula gastroyeyunal & $5(3,0 \%)$ & $5(1,2 \%)$ & $2(1,94 \%) \mathrm{p}<0,4$ \\
\hline Fístula duodenal & 0 & 1 & 1 \\
\hline Colección abdominal & 1 & 3 & 1 \\
\hline Fístula biliar & 0 & 0 & 1 \\
\hline Trombosis mesentérica & 0 & 1 & 0 \\
\hline Trombosis venosa profunda & 1 & 1 & 0 \\
\hline Hemorragia línea sutura & 1 & 4 & 0 \\
\hline Otros & 2 & 5 & 0 \\
\hline Total & $10(6,1 \%)$ & $20(4,7 \%)$ & $5(4,8 \%) \mathrm{p}>0,5$ \\
\hline
\end{tabular}


Hubo 14 reoperaciones (39\%) entre los 36 complicados ( $2 \%$ del total de pacientes operados). La distribución por sexo de los 36 pacientes con complicaciones mayores corresponde a 24 mujeres (67\%) y 12 hombres (33\%), con una edad promedio de 42,8 años, sin diferencia significativa comparado con el grupo no complicado.

El promedio anual de operaciones de bypass gástrico ha ido aumentando en forma constante, con un promedio anual, de los últimos cinco y medio años, de 124 pacientes/año. La estada hospitalaria para $93 \%$ de estos 684 pacientes fue de 6,5 días postoperado. Los pacientes con complicaciones tuvieron días de hospitalización variables.

\section{Discusión}

Los resultados del presente estudio demuestran que el bypass gástrico reseccional es una técnica alternativa en pacientes con obesidad mórbida que son sometidos a tratamiento quirúrgico, con cifras de morbilidad y mortalidad enteramente comparables a los del bypass gástrico sin gastrectomía. Los motivos por los cuales realizamos resección gástrica en nuestros pacientes han sido ampliamente discutidos previamente ${ }^{4,5}$ y se puede resumir en que, por una parte, no hay un aumento significativo de la morbilidad y mortalidad por el agregado de la gastrectomía y, por otra parte, no se conoce el riesgo de dejar un segmento gástrico excluido y muy difícil de evaluar para el resto de la vida del paciente. En la Tabla 3, se han seleccionado algunas publicaciones relevantes de la literatura extranjera referentes a la morbilidad y mortalidad del bypass gástrico abierto $3,6-17$, comparado al bypass gástrico reseccional que ha sido publicado en Estados Unidos de Norteamérica (EE.UU) y con nuestros datos. Se aprecia que hay una mortalidad operatoria cercana a $1 \%$, cifra mayor que nuestra mortalidad. La tasa de complicaciones y de fístula anastomótica, en general, coinciden con nuestras cifras. Llama la atención el cuidadoso análisis de las causas de mortalidad y morbilidad en estos pacientes que

Tabla 3. Resultados de morbimortalidad en algunas series importantes publicadas

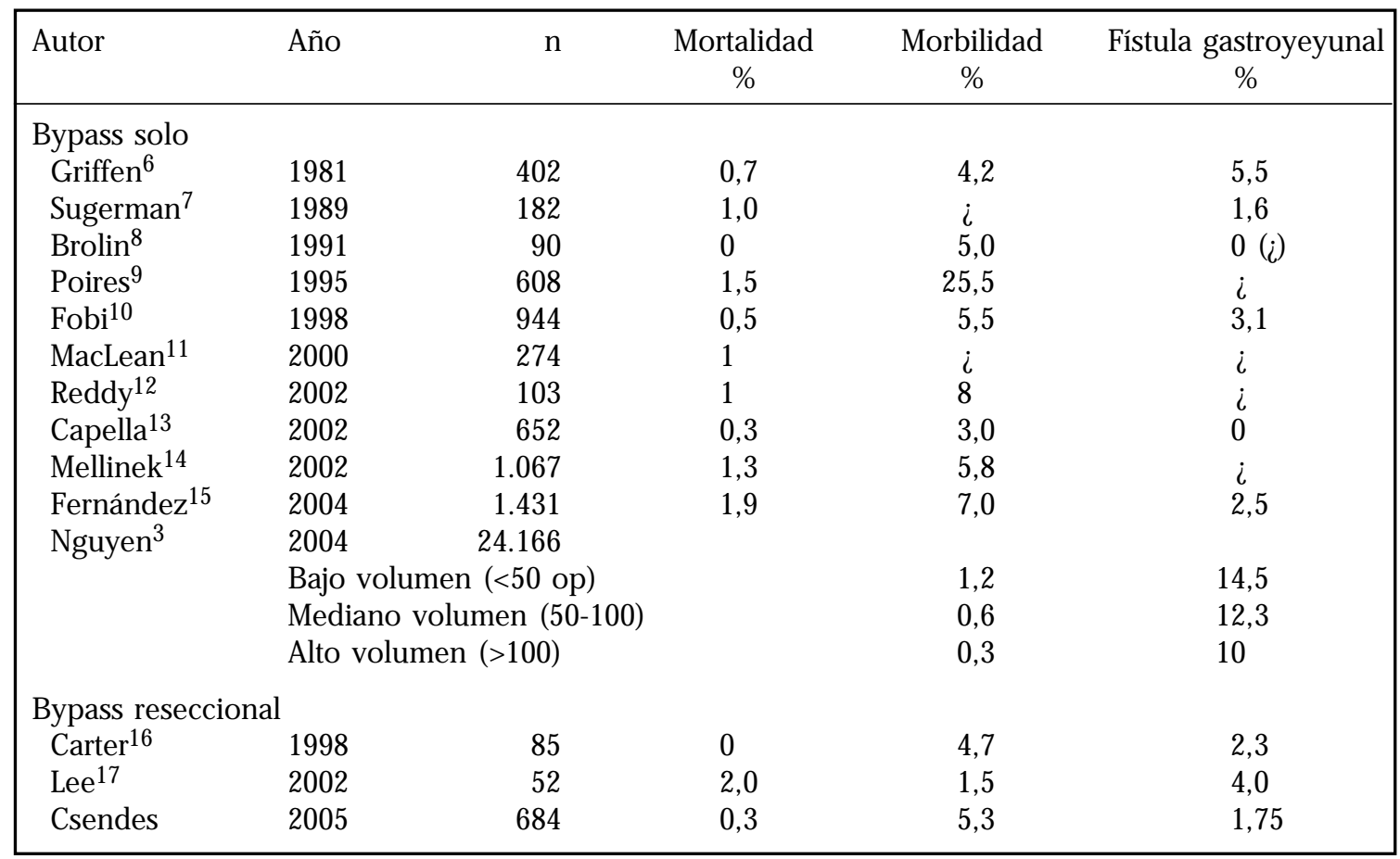


han reportado autores extranjeros. En algunos, se reportan esplenectomías de necesidad por lesiones intraoperatorias del bazo $8,10,13,17$. En dos publicaciones se señalan reoperaciones por cuerpos extraños dejados en la primera cirugía ${ }^{12,19}$. En dos publicaciones se mencionan cifras importantes de diarrea postoperatoria por Clostridium difficile ${ }^{13,17}$. La embolia pulmonar es siempre un problema latente y presente en estos pacientes. En un estudio referente a autopsias en diez fallecidos por cirugía bariátrica ${ }^{14}$, en ocho de ellos (80\%) se demostró la presencia de embolia pulmonar múltiple, silenciosa, que no se había diagnosticado previamente. Afortunadamente, en la presente serie no se ha presentado esta complicación.

Todos estos resultados recalcan que, por una parte, la cirugía bariátrica es una operación de gran envergadura, que se realiza en pacientes con alta complejidad médica y quirúrgica $\mathrm{y}$, por otra parte, que deben ser tratados por cirujanos expertos dedicados a cirugía gástrica. Llama la atención que en la serie de Reddy, de ciento tres operados, la única causa de mortalidad correspondió a un paciente que presentó una perforación aguda del estómago remanente y excluido del tránsito digestivo ${ }^{12}$. Recientemente, se han planteado dos hechos trascendentales en este tipo de cirugía: primero, que el volumen operatorio anual, es decir, el número de operaciones bariátricas por año en grupos con experiencia dedicados al tratamiento de esta patología, comparado con cirujanos con bajo volumen operatorio, tiene importantes repercusiones, tanto en la mortalidad operatoria como en la tasa de complicaciones. Nguyen et $\mathrm{al}^{3}$ evaluaron este parámetro, demostrando una mortalidad operatoria de 0,3\% para centros de alto volumen (más de 100 operaciones anuales) y una mortalidad cuatro veces mayor para centros de bajo volumen (menos de 50 operaciones anuales). Lo mismo ocurre para la tasa de complicaciones. Por otra parte, los estudios prospectivos y randomizados comparando bypass gástrico laparoscópico versus laparotómico ${ }^{18-20}$, han mostrado dos aspectos fundamentales: que la cirugía laparoscópica tiene menor morbilidad postoperatoria, con mejor evolución y bienestar del paciente pero, por otra parte, requiere de un importante esfuerzo del equipo quirúrgico para alcanzar la curva de aprendizaje para realizarla con éxito $^{3,19-21}$. Esta cirugía no puede ser producto de una improvisación, sino que requiere una constante preparación y dedicación del grupo quirúrgico. Está de más decir que el manejo de los pacientes con obesidad es multidisciplinario, con numerosos especialistas atendiéndolos. Este estudio solo pretende analizar un aspecto, que se refiere al riesgo quirúrgico. Como se aprecia, la cirugía bariátrica laparotómica tiene una mortalidad operatoria entre 0 y $1 \%$, una tasa de complicaciones postoperatorias de 3 a $20 \%$, una incidencia de fístula anastomótica entre 0 y $5 \%$ y una estada hospitalaria promedio entre cinco y siete días. A pesar de existir en Chile, varios grupos con experiencias variables, que realizan técnicas diferentes, no hay publicaciones precisas respecto al riesgo quirúrgico de cada grupo y en relación al volumen anual de las intervenciones. Nos parece que si el Ministerio de Salud decide implementar un programa de atención quirúrgica para pacientes con obesidad mórbida, se debiera cumplir un estricto protocolo de acreditación, tanto del centro quirúrgico postulante, como del grupo quirúrgico interesado en este tema, con realización de estadas de entrenamiento en centros calificados ${ }^{22}$. Nos parece que sólo centros de alto volumen quirúrgico debieran realizar esta cirugía bariátrica, para tener buenos resultados. Se podrá discutir el número anual mínimo de operaciones que debiera tener cada centro, pero nos parece que por lo menos debieran corresponder a más de 50 operaciones anuales, lo que representa una operación por semana. Es imprescindible que primero se realicen operaciones por vía laparotómica, para familiarizarse con la técnica. Posteriormente, la cirugía laparoscópica, a nuestro parecer, debiera realizarse sólo en algunos centros muy calificados. Tal como se ha expuesto en numerosas publicaciones internacionales ${ }^{3,17,19-21}$ la cirugía laparoscópica requiere de una larga y tediosa curva de aprendizaje y no todos logran reproducir los buenos resultados reportados por expertos ${ }^{19,21}$. Hay numerosos tópicos que requieren de una amplia discusión respecto a cuál técnica elegir: adolescentes, pacientes hiperobesos, operación abdominal mayor previa, etc. Cada grupo debe tener, a lo menos, experiencia con dos o tres procedimientos y esperamos con gran interés las publicaciones de otros grupos nacionales respecto a los resultados que han tenido respecto a la morbilidad y la mortalidad de las técnicas empleadas. 


\section{REFERENCIAS}

1. Christou NV, Sampales JS, Liberman M, Look D, Auger S, McLean APH et al. Surgery decreases long term mortality, morbidity and health care use in morbidly obese patients. Ann Surg 2004; 240: 416-24.

2. MacDonald KG, Long SD, Swenson MG. The gastric bypass reduces the progression and mortality of NIDDM. J Gastroint Surg 1997; 1: 213-20.

3. Nguyen NT, Paya M, Stevens M, Mavandadi SH, ZainABADI K, Wilson SE. The relationship between Hospital volume and outcome in bariatric surgery at academic centers. Ann Surg 2004; 240: 586-94.

4. Csendes A, Burdiles P, Díaz JC, Maluenda F, Burgos AM, Recio M ET AL. Resultados del tratamiento quirúrgico de la obesidad mórbida. Análisis de 180 pacientes. Rev Chil Cir 2002; 54: 3-9.

5. Csendes A, Burdiles P, Papapietro K, Díaz JC, Maluenda F, Burgos A et al. Results of gastric bypass plus resection of the distal excluded gastric segment in patients with morbid obesity. J Gastroint Surg 2005; 9: 121-31.

6. Griffen WO, Bivins BA, BeLl RM, Jackson KA. Gastric bypass for morbid obesity. World J Surg 1981; 5: 817-22.

7. Sugerman HG, Loudrey GL, Kelum JM. Weight loss with vertical banded gastroplasty and Roux-en-Y gastric bypass for morbid obesity with selective vs random assignment. Am J Surg 1989; 157: 93-102.

8. Broun RE, Keuler HA, Gorman JH, Cody RP. Long limb gastric bypass in the super obese: a prospective randomized trial. Ann Surg 1991; 215: 387-95.

9. Pories WJ, Swanson MS, MacDonald KG. Who would have thought it? An operation proves to be the most effective therapy for adult caused diabetes mellitus. Ann Surg 1995; 222: 339-52.

10. Fobi MAL, Lee H, Holness R, Cabrida D. Gastric bypass operation for obesity. World J Surg 1998; 22: 925-35.

11. MacLean LD, Rhode BM, Nohr CW. Late outcome of isolated gastric bypass. Ann Surg 2000; 231: 524-8.

12. Reddy RM, Ruker A, Mana D, Thomas R, Brems JJ. Open Roux-en-Y gastric bypass for the morbidly obese in the era of laparoscopy. Am J Surg 2002; 184: 611-6.

13. CAPELA JF, CAPELLA RF. An assessment of vertical banded gastroplasty Roux en Y gastric bypass for the treatment of morbid obesity. Am J Surg 2002; 183: 117-23.

14. Melinek J, Luvinsgton E, Cortina G, Fishbein MC. Autopsy findings following gastric bypass surgery for morbid obesity. Arch Pathol Lab Med 2002; 126: 1091-5.

15. Fernández AZ, De María EJ, Tichansky DS, Kelum JA, Worfe LG, Meador J et aL. Multivariate analysis of risk factors for death following gastric bypass for treatment of morbid obesity. Ann Surg 2004; 239: 698-703.

16. Curry TK, Carter PL, Porter CA, Watts DM. Resectional gastric bypass. Is a new alternative in morbid obesity. Am J Surg 1998; 175: 367-70.

17. Lee C, Cartes PL, Euott D, Mulenix PH, Eggebroten W, Porter C et al. An institutional experience with laparoscopic gastric bypass complications seen in the first year compared with open gastric bypass complications during the same period. Am J Surg 2002; 183: 533-8.

18. Lujan JA, Frutos D, Hernández Q, Liron R, Cuenca JR, VAlero G ET aL. Laparoscopic versus open gastric bypass in the treatment of morbid obesity. A randomized prospective study. Ann Surg 2004; 239: 433-7.

19. Nguyen NT, Goldman C, Rosenquist CJ, Arango A, Cole CJ, LeE SJ ET AL Laparoscopic versus open gastric bypass. A randomized study of outcomes, quality of life and costs. Ann Surg 2001; 234: 279-91.

20. Cottam DR, Mattar SG, Schauer PHR. Laparoscopic era of operations for morbid obesity. Arch Surg 2003; 138: 367-75.

21. Schauer PR, Ikrameddin S, Gourash W, Ramanattar $\mathrm{R}$, LuKETICH J. Outcomes after bypass laparoscopic Roux-en-Y gastric bypass for morbid obesity. Ann Surg 2000; 232; 515-20.

22. Carrasco F, Klaassen J, Papapietro K, Reyes E, Rodríguez L, Csendes A. et al. Propuesta y fundamentos para una norma de manejo quirúrgico del paciente obeso. Año 2004. Rev Méd Chile 2005; 133: 699-706. 